九州大学学術情報リポジトリ

Kyushu University Institutional Repository

\title{
TARSKI' S FIXED POINT THEOREM IN DEDEKIND CATEGORIES
}

Kawahara, Yasuo

Department of Informatics, Kyushu University

Honda, Kazumasa

Department of Informatics, Kyushu University

https://doi.org/10.5109/12599

出版情報: Bulletin of informatics and cybernetics. 38, pp.27-37，2006-12. Research Association of Statistical Sciences

バージョン:

権利関係 : 
TARSKI'S FIXED POINT THEOREM IN DEDEKIND CATEGORIES

by

Yasuo Kawahara and Kazumasa Honda

Reprinted from the Bulletin of Informatics and Cybernetics

Research Association of Statistical Sciences, Vol.38

FUKUOKA, JAPAN

2006 


\title{
TARSKI'S FIXED POINT THEOREM IN DEDEKIND CATEGORIES
}

\author{
By \\ Yasuo KAWAHARA $^{*}$ and Kazumasa HoNdA ${ }^{\dagger}$
}

\begin{abstract}
Tarski's fixed point theorem for complete lattices is a fundamental theorem in lattice theory and is very useful for computer science applications. This paper will show a general result in Dedekind categories, which extends the Tarski's theorem.
\end{abstract}

Key Words and Phrases: Dedekind category, Relation, Tarski's Fixed Point Theorem.

\section{Introduction}

Tarski's fixed point theorem (1955) proved a fixed point theorem: Every 1 function $f: L \rightarrow L$ on a complete lattice $(L, \leq)$ has the greatest fixed point. The key idea for the proof of this theorem is that the supremum of the set $T=\{x \in L \mid x \leq f(x)\}$ of all extending elements by $f$, is contained in $T$ and gives the desired fixed point. The Tarski's fixed point theorem has been applied in many fields of computer science and mathematics. Tarski (1941) also formulated algebras of binary relations, which is called "relation algebra" and established a modern foundation for relational methods in computer science and logic.

This paper aims to generalize Tarski's fixed point theorem (1955) in Dedekind categories. The Dedekind categories, initiated by Olivier and Serrato (1980) is a kind of relational categories, such as allegories by Freyd and Scedrov (1990). In order to restate Tarski's fixed point theorem in Dedekind categories we may need three basic ideas: transitive relations instead of orders, relational expressions for maximum and supremum (Cf. Schmidt and Ströhlein (1993) and Furusawa and Kahl (1998)), and a preservation condition, which generalizes the monotonicity of functions. This paper is organized as follows. In section 2 we will review the definition and the foundation of Dedekind categories. In section 3 we will study relational expression for maximum and supremum. In section 4 a preservation condition will be discussed. In section 5 the main theorem of the paper is proved and two examples are given.

\section{Dedekind Categories}

In this section we recall the definition of a kind of relation category which we will call Dedekind categories following Olivier and Serrato (1980). Dedekind categories are equivalent to locally complete division allegories introduced in Categories, allegories by

\footnotetext{
* Department of Informatics, Kyushu University 744, Fukuoka 819-0395 Japan. kawahara@i.kyushuu.ac.jp

† Department of Informatics, Kyushu University 744, Fukuoka 819-0395 Japan. honda@i.kyushu-u.ac.jp
} 
Freyd and Scedrov (1990).

Throughout this paper, a morphism $\alpha$ from an object $X$ into an object $Y$ in a Dedekind category (which will be defined below) will be denoted by a half arrow $\alpha$ : $X \rightarrow Y$, and the composite of a morphism $\alpha: X \rightarrow Y$ followed by a morphism $\beta: Y \rightarrow Z$ will be written as $\alpha \beta: X \neg Z$. Also we will denote the identity morphism on $X$ as $\operatorname{id}_{X}$.

Definition 2.1. A Dedekind category $\mathcal{D}$ is a category satisfying the following four conditions:

D1. [Complete Heyting Algebra] For all pairs of objects $X$ and $Y$ the hom-set $\mathcal{D}(X, Y)$ consisting of all morphisms of $X$ into $Y$ is a complete Heyting algebra (namely, a complete distributive lattice) with the least morphism $0_{X Y}$ and the greatest morphism $\nabla_{X Y}$. Its algebraic structure will be denoted by

$$
\mathcal{D}(X, Y)=\left(\mathcal{D}(X, Y), \sqsubseteq, \sqcup, \sqcap, 0_{X Y}, \nabla_{X Y}\right) .
$$

D2. [Converse] There is given a converse operation $\sharp: \mathcal{D}(X, Y) \rightarrow \mathcal{D}(Y, X)$. That is, for all morphisms $\alpha, \alpha^{\prime}: X \rightarrow Y, \beta: Y \rightarrow Z$, the following converse laws hold: (a) $(\alpha \beta)^{\sharp}=\beta^{\sharp} \alpha^{\sharp}$, (b) $\left(\alpha^{\sharp}\right)^{\sharp}=\alpha$, (c) If $\alpha \sqsubseteq \alpha^{\prime}$, then $\alpha^{\sharp} \sqsubseteq \alpha^{\prime \sharp}$.

D3. [Dedekind Formula] For all morphisms $\alpha: X \neg Y, \beta: Y \rightarrow Z$ and $\gamma: X \neg Z$ the Dedekind formula $\alpha \beta \sqcap \gamma \sqsubseteq \alpha\left(\beta \sqcap \alpha^{\sharp} \gamma\right)$ holds.

D4. [Residual Composition] For all morphisms $\alpha: X \neg Y$ and $\beta: Y \rightarrow Z$ the residual composite $\alpha \ominus \beta: X \neg Z$ is a morphism such that $\gamma \sqsubseteq \alpha \ominus \beta$ if and only if $\alpha^{\sharp} \gamma \sqsubseteq \beta$ for all morphisms $\gamma: X \rightarrow Z$.

A morphism $f: X \rightarrow Y$ such that $f^{\sharp} f \sqsubseteq \operatorname{id}_{Y}$ (univalent) and $\operatorname{id}_{X} \sqsubseteq f f^{\sharp}($ total) is called a total function and may be introduced as $f: X \rightarrow Y$. A total function $f: X \rightarrow Y$ is called a surjection if $f^{\sharp} f=\mathrm{id}_{Y}$. In what follows, the word relation is a synonym for morphism of a Dedekind category $\mathcal{D}$. A relation $\xi: X \neg X$ is called an order if it is reflexive $\left(\operatorname{id}_{X} \sqsubseteq \xi\right)$, transitive $(\xi \xi \sqsubseteq \xi)$ and antisymmetric $\left(\xi \sqcap \xi^{\sharp} \sqsubseteq \operatorname{id}_{X}\right)$. Note that $\xi$ is a preorder (namely, a reflexive and transitive relation) iff $\xi^{\sharp} \ominus \xi=\xi$.

In the rest of the section we list fundamental properties of residual composition, which will be needed in the latter sections.

Proposition 2.2. Let $\alpha, \alpha^{\prime}: X \rightarrow Y, \beta, \beta^{\prime}: Y \rightarrow Z, \gamma: Z \rightarrow W$ be relations in $\mathcal{D}$. Then the following holds:

(a) $(\alpha \ominus \beta) \gamma \sqsubseteq \alpha \ominus \beta \gamma$,

(b) If $\alpha \sqsubseteq \alpha^{\prime}$ and $\beta^{\prime} \sqsubseteq \beta$, then $\alpha^{\prime} \ominus \beta^{\prime} \sqsubseteq \alpha \ominus \beta$,

(c) $\alpha \ominus(\beta \ominus \gamma)=\alpha \beta \ominus \gamma$,

(d) $\alpha \sqsubseteq(\alpha \ominus \beta) \ominus \beta^{\sharp}$,

(e) $\left((\alpha \ominus \beta) \ominus \beta^{\sharp}\right) \ominus \beta=\alpha \ominus \beta$,

(f) If $f: U \rightarrow X$ is a total function, then $f \ominus \alpha=f \alpha$,

(g) If $g: V \rightarrow Y$ is a total function, then $(\alpha \ominus \beta) g^{\sharp}=\alpha \ominus \beta g^{\sharp}$, 
(h) If $f: U \rightarrow X$ and $g: V \rightarrow Y$ are total functions, then $f(\alpha \ominus \beta) g^{\sharp}=f \alpha \ominus \beta g^{\sharp}$,

(i) If $h: Y \rightarrow Z$ is a total function, then $\alpha h \ominus \gamma=\alpha \ominus h \gamma$.

Proof. (a) Since $\alpha^{\sharp}(\alpha \Theta \beta) \sqsubseteq \beta$ by definition of residual composition we have

$$
\alpha^{\sharp}(\alpha \ominus \beta) \gamma \sqsubseteq \beta \gamma,
$$

which is equivalent to $(\alpha \ominus \beta) \gamma \sqsubseteq \alpha \ominus \beta \gamma$.

(b) Assume $\alpha \sqsubseteq \alpha^{\prime}$ and $\beta \sqsubseteq \beta^{\prime}$. Then it follows that

$$
\alpha^{\sharp}\left(\alpha^{\prime} \ominus \beta^{\prime}\right) \sqsubseteq \alpha^{\prime \sharp}\left(\alpha^{\prime} \ominus \beta^{\prime}\right) \sqsubseteq \beta^{\prime} \sqsubseteq \beta
$$

and so $\alpha^{\prime} \ominus \beta^{\prime} \sqsubseteq \alpha \Theta \beta$.

(c) It follows at once from

$$
\begin{aligned}
\delta \sqsubseteq \alpha \ominus(\beta \ominus \gamma) & \Leftrightarrow \alpha^{\sharp} \sqsubseteq \beta \ominus \gamma & \{\ominus\} \\
& \Leftrightarrow \beta^{\sharp} \alpha^{\sharp} \sqsubseteq \gamma & \{\ominus\} \\
& \Leftrightarrow(\alpha \beta)^{\sharp} \delta \gamma & \{\text { Converse }\} \\
& \Leftrightarrow \delta \sqsubseteq \alpha \beta \ominus \gamma . & \{\ominus\}
\end{aligned}
$$

(d) It follows at once from

$$
(\alpha \odot \beta)^{\sharp} \alpha=\left(\alpha^{\sharp}(\alpha \odot \beta)\right)^{\sharp} \sqsubseteq \beta^{\sharp} .
$$

(e) First note that $\alpha \sqsubseteq(\alpha \ominus \beta) \ominus \beta^{\sharp}$ and $\alpha \ominus \beta \sqsubseteq\left((\alpha \ominus \beta) \ominus \beta^{\sharp}\right) \ominus \beta$ hold by (d). The first inclusion $\alpha \sqsubseteq(\alpha \ominus \beta) \ominus \beta^{\sharp}$ implies $\left((\alpha \ominus \beta) \ominus \beta^{\sharp}\right) \ominus \beta \sqsubseteq \alpha \ominus \beta$ by (b). Hence we have $\left((\alpha \ominus \beta) \ominus \beta^{\sharp}\right) \ominus \beta=\alpha \ominus \beta$.

(f) Assume that $f: U \rightarrow X$ is a total function. Then we have

$$
\begin{aligned}
\delta \sqsubseteq f \ominus \alpha & \Leftrightarrow f^{\sharp} \delta \sqsubseteq \alpha \quad\{\ominus\} \\
& \Leftrightarrow \delta \sqsubseteq f \alpha . \quad\left\{f^{\sharp} f \sqsubseteq \operatorname{id}_{X}, \operatorname{id}_{U} \sqsubseteq f f^{\sharp}\right\}
\end{aligned}
$$

(g) Assume that $g: V \rightarrow Y$ is a total function. Then we have

$$
\begin{array}{rlll}
(\alpha \ominus \beta) g^{\sharp} & \sqsubseteq \alpha \Theta \beta g^{\sharp} & \{(\mathrm{a})\} \\
& \sqsubseteq\left(\alpha \Theta \beta g^{\sharp}\right) g g^{\sharp} & \left\{\mathrm{id}_{V} \sqsubseteq g g^{\sharp}\right\} \\
& \sqsubseteq\left(\alpha \Theta \beta g^{\sharp} g\right) g^{\sharp} & \{(\mathrm{a})\} \\
& =(\alpha \Theta \beta) g^{\sharp} . & & \left\{(\mathrm{b}), g^{\sharp} g \sqsubseteq \mathrm{id}_{Y}\right\}
\end{array}
$$

(h) It is a direct corollary of (c), (f) and (g).

$$
\begin{array}{rlrl}
f(\alpha \Theta \beta) g^{\sharp} & =f\left(\alpha \Theta \beta g^{\sharp}\right) & & \{(\mathrm{g})\} \\
& =f \Theta\left(\alpha \Theta \beta g^{\sharp}\right) & \{(\mathrm{f})\} \\
& =f \alpha \Theta \beta g^{\sharp} . & & \{(\mathrm{c})\}
\end{array}
$$

(i) Assume that $h: Y \rightarrow Z$ is a total function. Then we have

$$
\alpha h \ominus \gamma=\alpha \ominus(h \ominus \gamma)=\alpha \ominus h \gamma
$$

by using (c) and (f). 


\section{Maximum and Supremum}

The concepts of upper-bounds, maximum and supremum are usually considered for subsets of an ordered set, and they do not always exist. Residual composition in Dedekind categories enables us to express them without assuming their existence. In particular, relational expressions for the concepts may deal with their existential extents. Schmidt and Ströhlein (1993) defined the concepts with composition and complement, and Furusawa and Kahl (1998) used quotients (namely, residues) instead of complement.

In this paper we will define the concepts using residual composition, which is equivalent to the quotients, because of the simplicity of symbolic treatments.

Definition 3.1. Let $\xi: X \rightarrow X$ and $\rho: V \rightarrow X$ be relations. The maximum relation $\max (\rho, \xi): V \rightarrow X$, the supremum relation $\sup (\rho, \xi): V \rightarrow X$, the minimum relation $\min (\rho, \xi): V \rightarrow X$, the infimum relation $\inf (\rho, \xi): V \rightarrow X$ for $\rho$ with respect to $\xi$ are defined as follows:

(a) $\max (\rho, \xi)=\rho \sqcap(\rho \ominus \xi)$,

(b) $\sup (\rho, \xi)=(\rho \ominus \xi) \sqcap\left((\rho \ominus \xi) \ominus \xi^{\sharp}\right)$,

(c) $\min (\rho, \xi)=\rho \sqcap\left(\rho \ominus \xi^{\sharp}\right)$,

(d) $\inf (\rho, \xi)=\left(\rho \ominus \xi^{\sharp}\right) \sqcap\left(\left(\rho \ominus \xi^{\sharp}\right) \ominus \xi\right)$.

In the above definition it is clear that $\sup (\rho, \xi)=\max \left(\rho \ominus \xi, \xi^{\sharp}\right)$. Also $\max (\rho, \xi) \sqsubseteq$ $\sup (\rho, \xi)$ always hold by Proposition $2.2(\mathrm{a})$.

Proposition 3.2. Let $\xi: X \rightarrow X$ and $\rho: V \rightarrow X$ be relations. Set $\theta=\xi \sqcap \xi^{\sharp}$. Then the following holds:

(a) $(\max (\rho, \xi))^{\sharp} \max (\rho, \xi) \sqsubseteq \theta$ and $(\sup (\rho, \xi))^{\sharp} \sup (\rho, \xi) \sqsubseteq \theta$,

(b) If $\xi$ is antisymmetric, then $\max (\rho, \xi)$ and $\sup (\rho, \xi)$ are univalent.

(c) If $\xi$ is antisymmetric and $\max (\rho, \xi)($ or $\sup (\rho, \xi))$ is total, then $\max (\rho, \xi)$ (or $\sup (\rho, \xi))$ is a total function,

(d) If $\xi$ is transitive, then $\sup (\rho, \xi) \theta=\sup (\rho, \xi)$ holds and $\sup (\rho, \xi)$ is difunctional.

Proof. (a) $\operatorname{As} \sup (\rho, \xi)=\max \left(\rho \ominus \xi, \xi^{\sharp}\right)$ it suffices to show the proof only for $\max (\rho, \xi)$.

$$
\begin{aligned}
(\max (\rho, \xi))^{\sharp} \max (\rho, \xi) & =\left(\rho^{\sharp} \sqcap(\rho \ominus \xi)^{\sharp}\right)(\rho \sqcap(\rho \ominus \xi)) & \\
& \sqsubseteq \rho^{\sharp}(\rho \ominus \xi) \sqcap\left(\rho^{\sharp}(\rho \ominus \xi)\right)^{\sharp} & \\
& \sqsubseteq \xi \sqcap \xi^{\sharp} . & \left\{\rho^{\sharp}(\rho \ominus \xi) \sqsubseteq \xi\right\}
\end{aligned}
$$

(b) It is direct from (a).

(c) It is a corollary of from (b). 
(d) Assume the transitivity $\xi \xi \sqsubseteq \xi$. Then we have

$$
\begin{array}{rlll}
\sup (\rho, \xi) \theta & \sqsubseteq(\rho \ominus \xi) \xi \sqcap\left((\rho \ominus \xi) \ominus \xi^{\sharp}\right) \xi^{\sharp} & & \left\{\theta=\xi \sqcap \xi^{\sharp}\right\} \\
& \sqsubseteq(\rho \ominus \xi \xi) \sqcap\left((\rho \ominus \xi) \ominus \xi^{\sharp} \xi^{\sharp}\right) & & \\
& \sqsubseteq(\rho \ominus \xi) \sqcap\left((\rho \ominus \xi) \ominus \xi^{\sharp}\right) & & \{\xi \xi \sqsubseteq \xi\} \\
& =\sup (\rho, \xi) & & \\
& \sqsubseteq \sup (\rho, \xi)(\sup (\rho, \xi))^{\sharp} \sup (\rho, \xi) & & \{\text { Dedekind Formula }\} \\
& \sqsubseteq \sup (\rho, \xi) \theta . & & \text { (a) }\}
\end{array}
$$

Generally it is difficult to compute maximum and supremum relations with respect to non antisymmetric relations. The next proposition gives a special formula to reduce supremum relations with respect to an order to supremum relations with respect to a preorder.

Proposition 3.3. Let $\eta: Y \rightarrow Y$ be an order and $q: X \rightarrow Y$ a surjection. Set $\xi=q \eta q^{\sharp}: X \rightarrow X$. Then the following holds:

(a) $\xi$ is a preorder with $\xi \sqcap \xi^{\sharp}=q q^{\sharp}$,

(b) $\sup (\rho, \xi)=\sup (\rho q, \eta) q^{\sharp}$ for each $\rho: V \rightarrow X$,

(c) If $\sup (\rho q, \eta)$ is total, then so is $\sup (\rho, \xi)$.

Proof. (a) It follows from $\operatorname{id}_{X} \sqsubseteq q q^{\sharp} \sqsubseteq q \eta q^{\sharp}, \xi \xi=q \eta q^{\sharp} q \eta q^{\sharp}=q \eta \eta q^{\sharp} \sqsubseteq q \eta q^{\sharp}=\xi$, and $\xi \sqcap \xi^{\sharp}=q \eta q^{\sharp} \sqcap q \eta^{\sharp} q^{\sharp}=q\left(\eta \sqcap \eta^{\sharp}\right) q^{\sharp}=q q^{\sharp}$.

(b) It is direct from a computation

$$
\begin{aligned}
\sup (\rho, \xi) & =\sup \left(\rho, q \eta q^{\sharp}\right) \\
& =\left(\rho \ominus q \eta q^{\sharp}\right) \sqcap\left(\left(\rho \ominus q \eta q^{\sharp}\right) \ominus q \eta^{\sharp} q^{\sharp}\right) \\
& =(\rho q \ominus \eta) q^{\sharp} \sqcap\left((\rho q \ominus \eta) q^{\sharp} q \ominus \eta^{\sharp}\right) q^{\sharp} \\
& =\left((\rho q \ominus \eta) \sqcap\left((\rho q \ominus \eta) \ominus \eta^{\sharp}\right)\right) q^{\sharp} \quad\left\{q^{\sharp} q=\operatorname{id}_{Y}\right\} \\
& =\sup (\rho q, \eta) q^{\sharp} .
\end{aligned}
$$

(c) Assume that $\sup (\rho q, \eta)$ is total. Then $\sup (\rho, \xi)=\sup (\rho q, \eta) q^{\sharp}$ is total, since $q^{\sharp} q=$ $\operatorname{id}_{Y}$.

\section{Preservation}

To state Tarski's fixed point theorem we have to define a preservation condition, which generalizes the monotonicity of functions. Although there may be a few candidates for the preservation, we prefer the following definition.

Definition 4.1. A relation $\alpha: X \rightarrow X$ preserves a relation $\xi: X \rightarrow X$ if $\alpha^{\sharp} \xi \alpha \sqsubseteq \xi$.

If a relation $\alpha: X \neg X$ preserves a reflexive and antisymmetric relation $\xi: X \neg X$, then the simple computation

$$
\alpha^{\sharp} \alpha=\alpha^{\sharp}\left(\xi \sqcap \xi^{\sharp}\right) \alpha \sqsubseteq \alpha^{\sharp} \xi \alpha \sqcap \alpha^{\sharp} \xi^{\sharp} \alpha \sqsubseteq \xi \sqcap \xi^{\sharp}=\operatorname{id}_{X}
$$


shows that $\alpha$ is univalent. This fact indicates that the preservation condition $\alpha^{\sharp} \xi \alpha \sqsubseteq \xi$ is relatively strong.

Also, if a relation $\alpha: X \rightarrow X$ preserves a transitive relation $\xi: X \rightarrow X$, then $\alpha \theta$ and $\theta \alpha \theta$ preserve $\xi$, where $\theta=\xi \sqcap \xi^{\sharp}$. For the preservation condition, $\alpha^{\sharp} \xi \alpha \sqsubseteq \xi$ implies

$$
\begin{array}{rlll}
(\theta \alpha \theta)^{\sharp} \xi(\theta \alpha \theta) & =\theta \alpha^{\sharp} \theta \xi \theta \alpha \theta & \left\{\theta^{\sharp}=\theta\right\} \\
& \sqsubseteq \theta \alpha^{\sharp} \xi \xi \xi \alpha \theta & \{\theta \sqsubseteq \xi\} \\
& \sqsubseteq \theta \alpha^{\sharp} \xi \alpha \theta & \{\xi \xi \sqsubseteq \xi\} \\
& \sqsubseteq \theta \xi \theta & & \left\{\alpha^{\sharp} \xi \alpha \sqsubseteq \xi\right\} \\
& \sqsubseteq \xi . &
\end{array}
$$

The following proposition shows that maximal relations preserving a preorder is easily determined.

Proposition 4.2. Assume that a total relation $\alpha: X \rightarrow X$ preserves a preorder $\xi: X \rightarrow X$. Set $\theta=\xi \sqcap \xi^{\sharp}$. Then the following holds:

(a) $\alpha \theta$ is maximal in the set of all relations on $X$ preserving $\xi$. In particular, $\theta \alpha \theta=\alpha \theta$ is valid.

(b) For a total relation $\beta: X \rightarrow X$ with $\beta \sqsubseteq \alpha \theta$, an identity $\beta \theta=\alpha \theta$ holds.

Proof. (a) We have stated that $\alpha \theta$ also preserves $\xi$. If $\alpha \theta \sqsubseteq \beta$ holds and $\beta$ preserves $\xi$, then

$$
\begin{array}{rlr}
\beta & \sqsubseteq \alpha \alpha^{\sharp} \beta & \left\{\operatorname{id}_{X} \sqsubseteq \alpha \alpha^{\sharp}\right\} \\
\sqsubseteq \alpha \theta \alpha^{\sharp} \theta \beta & \left\{\operatorname{id}_{X} \sqsubseteq \theta\right\} \\
\sqsubseteq \alpha \beta^{\sharp} \theta \beta & \{\alpha \theta \sqsubseteq \beta\} \\
& =\alpha \theta, & \left\{\beta^{\sharp} \theta \beta \sqsubseteq \theta\right\}
\end{array}
$$

which shows $\beta=\alpha \theta$.

(b) Assume that $\beta$ is total and $\beta \sqsubseteq \alpha \theta$ holds. Then $\beta$ also preserves $\xi$. By the virtue of (a) $\beta \theta$ is maximal among relations preserving $\xi$. Hence we have $\beta \theta \sqsubseteq \alpha \theta$ and $\beta \theta=\alpha \theta$.

The next lemma claims a simple property of transitive relations, which will be useful for the proof of the main theorem in Section 5 .

Lemma 4.3. If a relation $\alpha: X \rightarrow X$ preserves a transitive relation $\xi: X \rightarrow X$, then

$$
(\rho \ominus \xi) \alpha \sqsubseteq \rho \alpha \xi^{\sharp} \ominus \xi
$$

holds for all relations $\rho: V \rightarrow X$.

Proof. The inclusion follows from

$$
\begin{aligned}
\left(\rho \alpha \xi^{\sharp}\right)^{\sharp}(\rho \ominus \xi) \alpha & =\xi \alpha^{\sharp} \rho^{\sharp}(\rho \ominus \xi) \alpha & & \\
& \sqsubseteq \xi \alpha^{\sharp} \xi \alpha & & \left\{\rho^{\sharp}(\rho \ominus \xi) \sqsubseteq \xi\right\} \\
& \sqsubseteq \xi \xi & & \left\{\alpha^{\sharp} \xi \alpha \sqsubseteq \xi\right\} \\
& \sqsubseteq \xi . & & \{\xi \xi \sqsubseteq \xi\}
\end{aligned}
$$


The final proposition of the section shows that a relation preserving a preorder can be essentially reduced to a total function preserving an order.

Proposition 4.4. Let $\eta: Y \rightarrow Y$ be an order and $q: X \rightarrow Y$ a surjection. If a total relation $\alpha: X \rightarrow X$ preserves $\xi=q \eta q^{\sharp}: X \rightarrow X$, then the following holds:

(a) There exists a unique total function $h: Y \rightarrow Y$ such that $\alpha q=q h$,

(b) $h$ preserves $\eta$,

(c) $\nabla_{V X}\left(\alpha \xi^{\sharp} \sqcap \operatorname{id}_{X}\right) q=\nabla_{V Y}\left(h \eta^{\sharp} \sqcap \operatorname{id}_{Y}\right)$ for each object $V$.

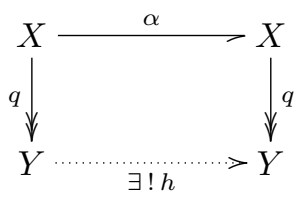

Proof. (a) If $\alpha q=q h$ for some total function $h$, then $h=q^{\sharp} \alpha q$ by $q^{\sharp} q=\mathrm{id}_{Y}$. Thus the uniqueness of $h$ is trivial. It suffices to show that $h=q^{\sharp} \alpha q$ is a total function and preserves $\eta$. The totality of $h$ follows from

$$
h h^{\sharp}=q^{\sharp} \alpha q q^{\sharp} \alpha^{\sharp} q \sqsupseteq q^{\sharp} \alpha \alpha^{\sharp} q \sqsupseteq q^{\sharp} q=\operatorname{id}_{Y} .
$$

Next we can show the univalency of $h$ as follows:

$$
\begin{aligned}
h^{\sharp} h & =q^{\sharp} \alpha^{\sharp} q q^{\sharp} \alpha q & & \left\{h=q^{\sharp} \alpha q\right\} \\
& =q^{\sharp} \alpha^{\sharp}\left(\xi \sqcap \xi^{\sharp}\right) \alpha q & & \left\{q q^{\sharp}=\xi \sqcap \xi^{\sharp}\right\} \\
& \sqsubseteq q^{\sharp} \alpha^{\sharp} \xi \alpha q \sqcap q^{\sharp} \alpha^{\sharp} \xi^{\sharp} \alpha q & & \{\text { Sub-distributive law }\} \\
& \sqsubseteq q^{\sharp} \xi q \sqcap q^{\sharp} \xi^{\sharp} q & & \left\{\alpha^{\sharp} \xi \alpha \sqsubseteq \xi\right\} \\
& =q^{\sharp} q \eta q^{\sharp} q \sqcap q^{\sharp} q \eta^{\sharp} q^{\sharp} q & & \left\{\xi=q \eta q^{\sharp}\right\} \\
& =\eta \sqcap \eta^{\sharp} & & \left\{q^{\sharp} q=\mathrm{id}_{Y}\right\} \\
& \sqsubseteq \mathrm{id}_{Y} . & & \left\{\eta \sqcap \eta^{\sharp} \sqsubseteq \mathrm{id}_{Y}\right\}
\end{aligned}
$$

The commutativity $\alpha q=q h$ is obtained from the totality of $\alpha q$, the univalency of $q h$ and an inclusion $\alpha q \sqsubseteq q q^{\sharp} \alpha q=q h$.

(b) The preservation condition $h^{\sharp} \eta h \sqsubseteq \eta$ holds since

$$
\begin{aligned}
h^{\sharp} \eta h & =\left(q^{\sharp} \alpha^{\sharp} q\right) \eta\left(q^{\sharp} \alpha q\right) & & \left\{h=q^{\sharp} \alpha q\right\} \\
& =q^{\sharp} \alpha^{\sharp} \xi \alpha q & & \left\{\xi=q \eta q^{\sharp}\right\} \\
& \sqsubseteq q^{\sharp} \xi q & & \left\{\alpha^{\sharp} \xi \alpha \sqsubseteq \xi\right\} \\
& =q^{\sharp} q \eta q^{\sharp} q & & \left\{\xi=q \eta q^{\sharp}\right\} \\
& =\eta . & & \left\{q^{\sharp} q=\mathrm{id}_{Y}\right\}
\end{aligned}
$$

Finally an identity $\nabla_{V X}\left(\alpha \xi^{\sharp} \sqcap \mathrm{id}_{X}\right) q=\nabla_{V Y}\left(h \eta^{\sharp} \sqcap \mathrm{id}_{Y}\right)$ is immediate from

$$
\begin{aligned}
\nabla_{V Y}\left(h \eta^{\sharp} \sqcap \operatorname{id}_{Y}\right) & =\nabla_{V Y}\left(h \eta^{\sharp} \sqcap \operatorname{id}_{Y}\right) q^{\sharp} q & & \left\{\operatorname{id}_{Y}=q^{\sharp} q\right\} \\
& =\nabla_{V Y}\left(h \eta^{\sharp} q^{\sharp} \sqcap q^{\sharp}\right) q & & \\
& =\nabla_{V Y} q^{\sharp}\left(q h \eta^{\sharp} q^{\sharp} \sqcap \operatorname{id}_{X}\right) q & & \{\text { Dedekind Formula }\} \\
& =\nabla_{V X}\left(\alpha q \eta^{\sharp} q^{\sharp} \sqcap \operatorname{id}_{X}\right) q & & \left\{\operatorname{id}_{X} \sqsubseteq q q^{\sharp}, q h=\alpha q\right\} \\
& =\nabla_{V X}\left(\alpha \xi^{\sharp} \sqcap \operatorname{id}_{X}\right) q . & & \left\{q \eta q^{\sharp}=\xi\right\}
\end{aligned}
$$




\section{Tarski's Fixed Point Theorem}

Then we can state the main result of the paper, which extends the Tarski's fixed point theorem (1955).

In this section we will use the following notation. For relations $\alpha: X \rightarrow X$ and $\xi: X \rightarrow X$ we set

$$
\tau=\nabla_{V X}\left(\alpha \xi^{\sharp} \sqcap \operatorname{id}_{X}\right), \quad \theta=\xi \sqcap \xi^{\sharp}, \quad \text { and } \quad \sigma=\nabla_{V X}(\alpha \theta \sqcap \theta)
$$

for an object $V$. The relation $\tau$ defined above just corresponds with the set of all extending elements $T$ stated in the introduction. Also, whenever $\xi$ is reflexive and antisymmetric, the relation $\sigma$ represents the set of all fixed points of $\alpha$.

We now state the main theorem of the paper. Although this theorem asserts inclusions, they turn out to be identities if $\xi$ is antisymmetric and $\sup (\tau, \xi)$ is total. This is the reason why this theorem is called Tarski's fixed point theorem.

TheOREM 5.1 TARski's FIXED POINT THEOREM. If a total relation $\alpha: X \rightarrow X$ preserves a transitive relation $\xi: X \rightarrow X$, then

$$
\sup (\tau, \xi) \alpha \sqsubseteq \sup (\tau, \xi) \alpha \theta=\max (\tau, \xi)=\sup (\tau, \xi) \sqsubseteq \max (\sigma, \xi) \sqsubseteq \sup (\sigma, \xi)
$$

holds.

Proof. Set $\tau_{0}=\sup (\tau, \xi)$. We have already seen that $\max (\tau, \xi) \sqsubseteq \sup (\tau, \xi)=\tau_{0}$ and $\max (\sigma, \xi) \sqsubseteq \sup (\sigma, \xi)$. It suffices to prove the following three statements:

(a) $\tau_{0} \alpha \sqsubseteq \max (\tau, \xi)$,

(b) $\tau_{0} \alpha \theta=\tau_{0}$,

(c) $\tau_{0} \sqsubseteq \max (\tau, \xi)$ and $\tau_{0} \sqsubseteq \max (\sigma, \xi)$.

(a) By the definition of $\tau$ we have

$$
\tau=\nabla_{V X}\left(\alpha \xi^{\sharp} \sqcap \operatorname{id}_{X}\right)\left(\alpha \xi^{\sharp} \sqcap \operatorname{id}_{X}\right) \sqsubseteq \tau \alpha \xi^{\sharp}
$$

and so $\tau \sqsubseteq \tau \alpha \xi^{\sharp}$. Thus, to show $\tau_{0} \alpha \sqsubseteq \max (\tau, \xi)$ we have to $\tau_{0} \alpha \sqsubseteq \tau \ominus \xi$ and $\tau_{0} \alpha \sqsubseteq \tau$. The first inclusion $\tau_{0} \alpha \sqsubseteq \tau \ominus \xi$ follows from a computation

$$
\begin{array}{rlll}
\tau_{0} \alpha & \sqsubseteq(\tau \ominus \xi) \alpha & & \{\sup (\tau, \xi) \sqsubseteq \tau \ominus \xi\} \\
& \sqsubseteq \tau \alpha \xi^{\sharp} \ominus \xi & & \{\operatorname{Lemma} 4.3\} \\
& \sqsubseteq \tau \ominus \xi . & & \left\{\tau \sqsubseteq \tau \alpha \xi^{\sharp}\right\}
\end{array}
$$

On the other hand, since $\tau_{0} \sqsubseteq(\tau \ominus \xi) \ominus \xi^{\sharp}$ by the definition of $\tau_{0}$, we have

$$
\begin{aligned}
\tau \ominus \xi & =\left((\tau \ominus \xi) \ominus \xi^{\sharp}\right) \ominus \xi \\
& \sqsubseteq \tau_{0} \ominus \xi .
\end{aligned} \quad\left\{\tau_{0} \sqsubseteq(\tau \ominus \xi) \ominus \xi^{\sharp}\right\}
$$


This inclusion and the first one jointly imply an inclusion $\tau_{0} \alpha \sqsubseteq \tau_{0} \ominus \xi$. Hence the second inclusion $\tau_{0} \alpha \sqsubseteq \tau$ follows from

$$
\begin{aligned}
\tau_{0} \alpha & =\left(\tau_{0} \ominus \xi\right) \sqcap \tau_{0} \alpha & & \left\{\tau_{0} \alpha \sqsubseteq \tau_{0} \ominus \xi\right\} \\
& \sqsubseteq \tau_{0} \alpha\left(\alpha^{\sharp} \tau_{0}^{\sharp}\left(\tau_{0} \ominus \xi\right) \sqcap \mathrm{id}_{X}\right) & & \{\text { Dedekind Formula }\} \\
& \sqsubseteq \nabla_{V X}\left(\alpha^{\sharp} \xi \sqcap \mathrm{id}_{X}\right) & & \left\{\tau_{0} \alpha \sqsubseteq \nabla_{V X}, \tau_{0}^{\sharp}\left(\tau_{0} \ominus \xi\right) \sqsubseteq \xi\right\} \\
& =\nabla_{V X}\left(\xi^{\sharp} \alpha \sqcap \mathrm{id}_{X}\right) & & \left\{\alpha^{\sharp} \xi \sqcap \mathrm{id}_{X} \sqsubseteq \mathrm{id}_{X}\right\} \\
& \sqsubseteq \nabla_{V X}\left(\alpha \xi^{\sharp} \sqcap \mathrm{id}_{X}\right) & & \left\{\alpha^{\sharp} \xi^{\sharp} \alpha \sqsubseteq \xi^{\sharp}, \mathrm{id}_{X} \sqsubseteq \alpha \alpha^{\sharp}\right\} \\
& =\tau . & &
\end{aligned}
$$

(b) The inclusion $\tau_{0} \alpha \sqsubseteq \max (\tau, \xi) \sqsubseteq \tau_{0}$ is known in (a). Since $\tau_{0} \theta=\tau_{0}$ by Proposition 3.2 (c), we have $\tau_{0} \alpha \theta \sqsubseteq \tau_{0}$. On the other hand it follows that

$$
\begin{aligned}
\tau_{0} & \sqsubseteq \tau_{0} \tau_{0}^{\sharp} \tau_{0} & & \{\text { Dedekind Formula }\} \\
& \sqsubseteq \tau_{0} \alpha \alpha^{\sharp} \tau_{0}^{\sharp} \tau_{0} & & \left\{\operatorname{id}_{X} \sqsubseteq \alpha \alpha^{\sharp}\right\} \\
& \sqsubseteq \tau_{0} \alpha \tau_{0}^{\sharp} \tau_{0} & & \left\{\tau_{0} \alpha \sqsubseteq \tau_{0}\right\} \\
& \sqsubseteq \tau_{0} \alpha \theta . & & \left\{\tau_{0}^{\sharp} \tau_{0} \sqsubseteq \theta\right\}
\end{aligned}
$$

Therefore we have proved $\tau_{0} \alpha \theta=\tau_{0}$.

(c) First we will show $\tau_{0} \sqsubseteq \sigma \sqsubseteq \tau$. An inclusion $\tau_{0} \sqsubseteq \sigma$ follows from

$$
\begin{aligned}
& \tau_{0}=\tau_{0} \alpha \theta \sqcap \tau_{0} \quad\left\{(\mathrm{~b}) \tau_{0}=\tau_{0} \alpha \theta\right\} \\
& \sqsubseteq \tau_{0}\left(\alpha \theta \sqcap \tau_{0}^{\sharp} \tau_{0}\right) \quad\{\text { Dedekind Formula } \\
& \sqsubseteq \nabla_{V X}(\alpha \theta \sqcap \theta) \quad\left\{\tau_{0} \sqsubseteq \nabla_{V X}, \tau_{0}^{\sharp} \tau_{0} \sqsubseteq \theta\right\} \\
& =\sigma \text {. }
\end{aligned}
$$

Also $\sigma \sqsubseteq \tau$ follows from

$$
\begin{aligned}
\sigma & =\nabla_{V X}(\alpha \theta \sqcap \theta) & & \\
& \sqsubseteq \nabla_{V X}\left(\alpha \xi^{\sharp} \sqcap \xi\right) & & \left\{\theta=\xi \sqcap \xi^{\sharp}\right\} \\
& \sqsubseteq \nabla_{V X} \xi\left(\xi^{\sharp} \alpha \xi^{\sharp} \sqcap \mathrm{id}_{X}\right) & & \{\text { Dedekind Formula }\} \\
& \sqsubseteq \nabla_{V X} \xi\left(\alpha \xi^{\sharp} \xi^{\sharp} \sqcap \mathrm{id}_{X}\right) & & \left\{\alpha^{\sharp} \xi^{\sharp} \alpha \sqsubseteq \xi^{\sharp}, \mathrm{id}_{X} \sqsubseteq \alpha \alpha^{\sharp}\right\} \\
& \sqsubseteq \nabla_{V X}\left(\alpha \xi^{\sharp} \sqcap \mathrm{id}_{X}\right) & & \{\xi \xi \sqsubseteq \xi\} \\
& =\tau . & &
\end{aligned}
$$

Hence $\tau_{0} \sqsubseteq \sigma \sqsubseteq \tau$ holds. Since $\tau_{0} \sqsubseteq \tau \ominus \xi$ by the definition of $\tau_{0}$ we have

$$
\tau_{0} \sqsubseteq \tau \ominus \xi \sqsubseteq \sigma \ominus \xi,
$$

which implies $\tau_{0} \sqsubseteq \max (\tau, \xi)$ and $\tau_{0} \sqsubseteq \max (\sigma, \xi)$. This completes the proof.

A few interesting corollaries are direct from the main theorem 5.1.

Corollary 5.2. If a total relation $\alpha: X \rightarrow X$ preserves a transitive and antisymmetric relation $\xi: X \rightarrow X$, then

$$
\sup (\tau, \xi) \alpha=\sup (\tau, \xi) \alpha \theta=\max (\tau, \xi)=\sup (\tau, \xi)
$$

holds. 
Proof. Set $\tau_{0}=\sup (\tau, \xi)$. We have already seen $\tau_{0} \alpha \sqsubseteq \max (\tau, \xi)=\tau_{0}=\tau_{0} \alpha \theta$ in Theorem 5.1. It follows from the antisymmetry $\theta \sqsubseteq \operatorname{id}_{X}$ of $\xi$ that $\tau_{0} \alpha \theta \sqsubseteq \tau_{0} \alpha$. Hence $\tau_{0} \alpha=\max (\tau, \xi)=\tau_{0}=\tau_{0} \alpha \theta$ holds.

Corollary 5.3. If a total relation $\alpha: X \rightarrow X$ preserves a transitive relation $\xi: X \rightarrow X$ and if $\sup (\tau, \xi)$ is total, then

$$
\sup (\tau, \xi) \alpha \theta=\max (\tau, \xi)=\sup (\tau, \xi)=\max (\sigma, \xi)=\sup (\sigma, \xi)
$$

holds.

Proof. Set $\tau_{0}=\sup (\tau, \xi)$ and $\sigma_{0}=\sup (\sigma, \xi)$. We have already seen $\tau_{0} \alpha \theta \sqsubseteq \sup (\sigma, \xi)=$ $\sigma_{0}$ in Theorem 5.1. It suffices to prove $\sigma_{0} \sqsubseteq \tau_{0} \alpha \theta$ :

$$
\begin{aligned}
& \sigma_{0} \sqsubseteq \tau_{0} \alpha\left(\tau_{0} \alpha\right)^{\sharp} \sigma_{0} \quad\left\{\tau_{0}, \alpha: \text { total }\right\} \\
& \sqsubseteq \tau_{0} \alpha \sigma_{0}^{\sharp} \sigma_{0} \quad\left\{\text { Theorem 5.1: } \tau_{0} \alpha \sqsubseteq \sigma_{0}\right\} \\
& \sqsubseteq \tau_{0} \alpha \theta . \quad\left\{\text { Proposition 3.2(a): } \sigma_{0}^{\sharp} \sigma_{0} \sqsubseteq \theta\right\}
\end{aligned}
$$

Corollary 5.4. Assume that all nonzero relations $\rho: V \rightarrow X$ are total. If a total relation $\alpha: X \rightarrow X$ preserves a transitive relation $\xi: X \rightarrow X$ and if $\sup (\tau, \xi) \neq 0_{V X}$, then

$$
\sup (\tau, \xi) \alpha \theta=\max (\tau, \xi)=\sup (\tau, \xi)=\max (\sigma, \xi)=\sup (\sigma, \xi)
$$

holds.

Finally we will list two examples. First we show an example that the set of extending elements and the set of fixpoints of $f$ are not always identical.

ExAmple 5.5. Let $X$ be the set of all reals, $\xi$ the order ('less than or equal to') on $X$ and $f(x)=x^{3}$ a total function on X. Set $I=\{*\}$. Then it is readily seen that $\tau=\nabla_{I X}\left(f \xi^{\sharp} \sqcap \mathrm{id}_{X}\right)=\{(*, x) \mid-1 \leq x \leq 0$ or $1 \leq x\}, \tau \ominus \xi=0_{I X}$ (the empty relation), $\sigma=\nabla_{I X}\left(f \sqcap \operatorname{id}_{X}\right)=\{(*,-1),(*, 0),(*, 1)\}$, and $\max (\sigma, \xi)=\sup (\sigma, \xi)=\{(*, 1)\}$. Hence an identity $\sup (\tau, \xi)=\max (\sigma, \xi)$ does not always hold (Cf. Theorem 5.1).

In general, it is difficult to find fixpoints for relations. The results of Proposition 3.3, 4.4 and Corollary 5.3 enable us to compute fixpoints for relations under relevant conditions. The following is one of such examples.

ExAmple 5.6. Let $X=\mathbb{C}-\{0\}$ be the set of all nonzero complex numbers, $Y=\mathbb{R}^{+}$ the set of all positive reals, $\eta: Y \rightarrow Y$ the order ('less than or equal to') on $Y$, and $q: X \rightarrow Y$ a surjection with $q(z)=|z|$ for all $z \in X$. Then $\xi=q \eta q^{\sharp}$ is a preorder on $X$ with $\xi=\{(z, w) \in X \times X|| z|\leq| w \mid\}$. Let $\alpha: X \neg X$ be a relation defined as

$$
\alpha=\left\{(z, w) \in X \times X \mid \sqrt{|z|} w=z e^{i \theta} \text { for some } \theta \text { with } \pi / 2 \leq \theta \leq \pi\right\} .
$$

It is trivial that $\alpha$ is total and preserves $\xi$. Also $\alpha$ has no fixed point, in other word, there is no complex number $z \in X$ with $(z, z) \in \alpha$. By Proposition 4.4 there exists a 
unique total function $h: Y \rightarrow Y$ such that $\alpha q=q h$. It is trivial that $h(r)=\sqrt{r}$ for all positive reals $r$. Moreover, setting $\tau_{\alpha}=\nabla_{I X}\left(\alpha \xi^{\sharp} \sqcap \operatorname{id}_{X}\right)$ and $\tau_{h}=\nabla_{I Y}\left(h \eta^{\sharp} \sqcap \operatorname{id}_{Y}\right)$, we have

$$
\sup \left(\tau_{h}, \eta\right)=\max \left(\tau_{h}, \eta\right)=\{(*, 1)\}
$$

and so

$$
\sup \left(\tau_{\alpha}, \xi\right)=\max \left(\tau_{\alpha}, \xi\right)=\{(*, z)|| z \mid=1\}
$$

by Proposition 3.3, 4.4 and Corollary 5.3.

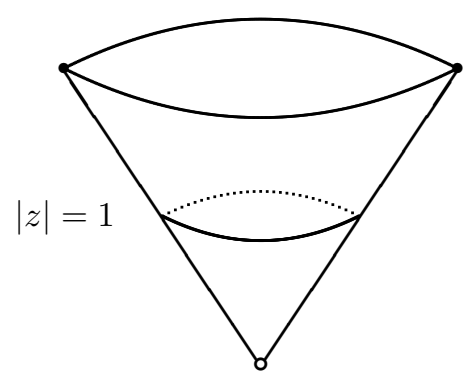

$$
X=\mathbb{C}-\{0\}
$$

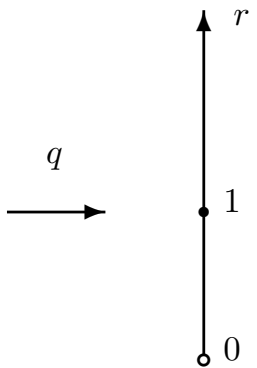

$Y=\mathbb{R}^{+}$

\section{Conclusion}

This paper presented Tarski's fixed point theorem in Dedekind categories to show that relational methods could give more general versions than the original theorem. Moreover a concrete example of relational version was illustrated. To find effective applications of the relational Tarski's theorem is an interesting future work.

\section{References}

Freyd, P. and Scedrov, A. (1990). Categories, allegories, North-Holland, Amsterdam.

Furusawa, H. and Kahl, W. (1998). A study on symmetric quotients, Technical Report Nr. 1998-06, Fakultät für Informatik, Univesität der Bundeswehr München.

Olivier, J. and Serrato, D. (1980). Catégories de Dedekind. Morphismes dans les Catégories de Schröder, C. R. Acad. Sci. Paris 260 939-941.

Schmidt, G. and Ströhlein, T. (1993). Relations and graphs - Discrete Mathematics for Computer Science -, Springer-Verlag, Berlin.

Tarski, A. (1941). On the calculus of relations, J. Symbolic Logic 6 73-89.

Tarski, A. (1955). A lattice theoretical fixpoint theorem and its applications, Pacific J. Math. 5 285-309.

Received October 12, 2004

Revised September 16, 2005 